\title{
Personality and Perception of Stigma in Psychiatric Patients with DEPRESSIVE DISORDERS
}

\author{
L. Borecki1 ${ }^{1}$ A. Gozdzik-Zelazny ${ }^{1}$, M. Pokorski1,2 \\ ${ }^{1}$ Institute of Psychology, Opole University, Opole, Poland; ${ }^{2}$ Medical Research Center, Polish Academy of Sciences, Warsaw, Poland
}

\begin{abstract}
Objective: The study seeks to determine the relationships between neuroticism and extroversion, on the one side, and the perception of various dimensions of social stigma, on the other, in psychiatric in-patients with depressive disorders, such as depressive episodes, or mood and anxiety disorders with the presence of depressive symptoms.

Material and methods: A total of 72 patients were examined in the study. Twenty four of them (F/M 12/12; age range 42-65 years) were used for assessing the reliability of a depression stigma questionnaire (DSQ) created specifically for this study. The remaining 48 patients $(\mathrm{F} / \mathrm{M}-31 / 17$; age range $17-74$ years) were then surveyed with the DSQ and other psychometric tools employed in the study. Self-reported data on the self-stigma, perceived stigma, public (mirrored) stigma, secrecy, treatment stigma, and the levels of neuroticism, extroversion, and depression were collected.

Results: The study demonstrates that treatment stigma and secrecy were the most frequent aspects of stigma experienced by the patients. Secrecy correlated negatively with age and positively with the education level. There were correlations between neuroticism and four of the stigma dimensions: treatment stigma, secrecy, perceived stigma, and self-stigma. Extroversion, in turn, correlated with perceived stigma, public stigma, and self-stigma.

Conclusions: The study confirms the relevance of personality resources in the perception and coping with stigma and points to secrecy as a major underlying factor in these processes. In light of the present findings, stigma experienced by the patient is not only related to the external indicators of social stigmatization and the state of depression, but also to the personal features of an individual.
\end{abstract}

Key words: stigma, mental illness, depression, personality, neuroticism, extroversion

\section{INTRODUCTION}

Mental illness brings social stereotypes, prejudices, discrimination, rejection, and loss of the social status. Stereotypes related to particular mental illnesses differ slightly in content, but are all based on the same false assumptions that people with psychiatric disorders are, in general, dangerous, incompetent, careless, unpre- dictable, aggressive, hostile, etc [1]. Additionally, in case of depression, personal responsibility for the disease, lack of will to recover and of the individual's control of a depressive condition all are attributed to the patient. Due to insufficient mental health literacy, resulting in stereotypes and feelings of fear and anger, people with psychiatric disorders become victims of prejudices held by almost every social group [2]. Those prejudiced beliefs are of main significance in the most easily observed dimension of mental illness, stigma - discrimination, which is defined in this article as a hostile behavioral expression of one's prejudice [3]; the expression varying in its form from offensive language, backbiting, or social exclusion to job and accommodation refusal, or court injustice [4].

Although stigma of mental illness has been evaluated since the 1960s, it is only recently that the patient's perception and coping with stigma became a subject of the systematic psychological investigation. Perceiving stigmatizing social attitudes and behaviors may influence the depressive patient's recovery process by his resigning from seeking medical or psychological help [5], drug adherence, or by treatment discontinuation $[6,7]$. Yet, not all patients report perceiving stigma and not all of them perceive it in the same way and with the same power. Also, they may choose different ways of coping [8]. An explanation of this may be that awareness of stereotypes, prejudices, and discrimination are stressors to which one should respond using his individual stress coping resources [9].

In present study we set out to investigate whether personality traits contributing to stress coping, such as neuroticism and extroversion, are associated with chosen factors of stigma perception and coping in patients with depressive disorders. Also, we wanted to assess the force of particular stigma factors and explore demographical differences. We chose five factors of stigma displayed by depressive psychiatric patients: 1) treatment stigma, which is a belief that negative attitudes toward receiving psychiatric treatment exist in society; 2) secrecy, which is a coping strategy based on keeping one's condition in secret; 3) perceived stigma, which is a belief that negative attitudes toward depressive patients exist in society; 4) public or mirrored stig$\mathrm{ma}$, which is the patient's internalized prejudices against other patients; and 5) self-stigma, which consists of negative attitudes toward oneself arising from internalized perceived stereotypes and prejudice. We hypothesized that high levels of the stigma dimen- 
sions above outlined could relate with neuroticism and extroversion in depressive patients.

\section{Material And Methods}

\section{SuBJECTS}

The study was performed in accordance with the Declaration of Helsinki for Human Research and the study protocol was approved by an institutional Research Review Committee. Patients, who were enrolled into the study, gave informed consent after being informed about the aim and method of the study. No incentive was offered for enrolment into the study.

The study was carried out on a total of 72 psychiatric in-patients with depressive disorders. Twenty four of them $(\mathrm{F} / \mathrm{M}-12 / 12)$ of the mean age of $54 \pm 6$ (range 42-65 years) were used for assessing the reliability of a Depression Stigma Questionnaire (DSQ) created specifically for this study (see details below). The remaining 48 patients $(\mathrm{F} / \mathrm{M}-31 / 17)$ of the mean age of $55 \pm 10$ (range 17-74 years) were then surveyed with the DSQ and other psychometric tools employed in the study, regarding the perception of stigmatization. Since there were no obvious differences in the score results depending on gender, all data were pooled and analyzed together. The patients of the latter group were diagnosed with depressive episodes $(12.5 \%$ cases), major depressive disorder $(45.8 \%)$, organic mood disorder, $(29.2 \%)$, and generalized anxiety disorder $(12.5 \%)$; the latter two with the predominance of depressive symptoms, and were hospitalized in psychiatric wards of hospitals in the cities of Warta, Opole, Rybnik, and Lubliniec in Poland at the time of the survey.

Duration of disease in the group of subjects used for the verification of questionnaire's reliability was approximately $1-5$ years in 8 participants, 6-10 years in 4 participants, $11-15$ years in 3 participants, 30-35 or more in 2 participants, and in the remaining 7 participants the data were not provided. Duration of disease in the main experimental group of patients, surveyed for the perception of stigmatization, was 1-5 years in 23 participants, 6-10 years in 8 participants, 11-15 years in 7 participants, 16-20 years in 3 participants, 21-25 years in 1 participant, $26-30$ years in 3 participants, 31-35 years in 1 participants, and in the remaining 2 participants the data were not provided. In this experimental group, Beck's Depression Inventory scores showed mild-to-moderate depression in $27.1 \%$ patients, moderate-to-severe depression in $60.4 \%$ patients, and severe depression $4.2 \%$. No depressive symptoms were declared by patients in $8.3 \%$ cases.

About $90 \%$ of the participants in both groups currently used antidepressive medicines and were subjected to active psychobehavioral therapy. There were no major differences between the two groups in terms of social or economic standings; about $45 \%$ of the participants were married, 60-70\% were pensioners or retirees of poor financial standing at about one-third of the average income in Poland. In the main 48-patient group studied for the perception of stigmatization, there were 26 single and 22 married persons. Concerning education, $14(29 \%)$ participants had primary, 12
(25\%) participants had vocational, 16 (33\%) participants had secondary (high school), and $6(13 \%)$ had college or university education. Thirty four of the 48 lived with significant others, i.e., the family in the same dwelling, and 14 persons lived lonely.

\section{PSYCHOMETRIC TOOLS}

All surveys were conducted during the patient's stay in a psychiatric hospital ward. Depressive symptoms were measured with Beck's Depression Inventory. To measure neuroticism and extroversion/introversion adequate scales from Costa and McCrae's NEO-FFI in the 1998 Polish version by Zawadzki et al [10] were used. Factors of stigma were measured using the above-mentioned DSQ. This questionnaire was based on the factors of Self-stigma and Perceived stigma presented by Corrigan and Kleinlein [5] and Secrecy, Public or mirrored stigma, and Treatment stigma presented by Kanter et al [11] in their Depression SelfStigma Scale. Each of DSQ subscales consists of 6 items. All subscales were tested for reliability using Cronbach's alpha in a sample of 24 psychiatric patients with depressive disorders. The reliability indicators were satisfactory and are presented in Table 1. The answers were marked on 5-degree Likert-type scale and scored as follows: 0 - completely disagree, 1 - disagree, 2 - do not know, 3 - agree, and 4 - completely agree. A higher score indicated a higher level of a stigma factor. There were no reversed questions and an overall score was not tallied. The subscales of DSQ did not correlated with one another in the preliminary part of the study devoted to the validation of the DSQ, although they did correlate in the main experimental part of the study (see Results).

All questionnaires were anonymous. Participants were surveyed individually or in small groups, and were assisted by the examiner. The time to fill out the questionnaires was not limited and, on average, amounted to 30-45 $\mathrm{min}$.

Table 1. Cronbach's alpha for DSQ subscales.

\begin{tabular}{lccc}
\hline Scale & $\begin{array}{c}\text { Number } \\
\text { of items }\end{array}$ & $\begin{array}{c}\text { Cronbach's } \\
\text { alpha }\end{array}$ & $\begin{array}{c}\text { Mean score } \\
\pm \text { SE }\end{array}$ \\
\hline Treatment stigma & 6 & 0.613 & $20.4 \pm 0.8$ \\
Secrecy & 6 & 0.794 & $20.5 \pm 1.0$ \\
Perceived stigma & 6 & 0.673 & $18.8 \pm 0.8$ \\
Public stigma & 6 & 0.835 & $14.9 \pm 1.1$ \\
Self-stigma & 6 & 0.825 & $14.6 \pm 1.0$
\end{tabular}

Data are presented as means \pm SE. Fisher's least significant differences (LSD) test was used for comparing mean raw scores of stigma factors. An unpaired t-test and Mann Whitney U test were used, as appropriate, for comparison between two groups. Pearson's correlations between neuroticism, extroversion, and the factors of stigma evaluated was computed, Kendall's tau $(\tau)$ coefficient was a statistic used to measure the association between the two measured quantities of education and secrecy. 


\section{RESULTS}

Force of STIGMA FACTORS

Comparison of the mean scores for stigma factors in depressive patients shows that treatment stigma and

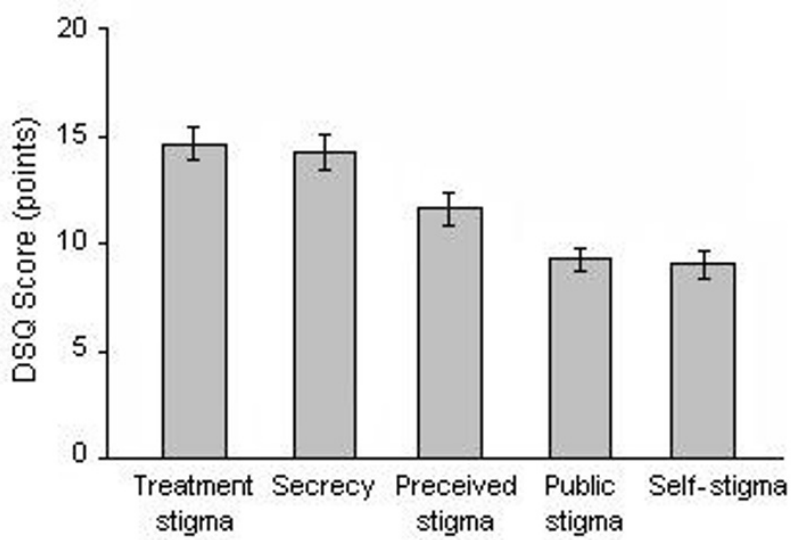

Fig. 1. Force of stigma factors.

Table 2. Mean differences in raw scores describing the force of stigma factors.

\begin{tabular}{llll}
\hline Factor 1 & Factor 2 & $\begin{array}{l}\text { Difference } \\
(1-2)\end{array}$ & $\mathrm{P}$ \\
\hline Treatment stigma & Secrecy & $\mathbf{0 . 4} \pm \mathbf{0 . 7}$ & $\mathbf{0 . 5 0 5}$ \\
& $\begin{array}{l}\text { Perceived stigma } \\
3.0 \pm 0.6\end{array}$ & 0.001 \\
& Public stigma & $5.4 \pm 0.8$ & 0.001 \\
& Self-stigma & $5.6 \pm 0.7$ & 0.001 \\
\hline Secrecy & Perceived stigma & $2.6 \pm 0.9$ & 0.005 \\
& Public stigma & $4.9 \pm 0.9$ & 0.001 \\
& Self-stigma & $5.2 \pm 0.9$ & 0.001 \\
\hline Perceived stigma & Public stigma & $2.3 \pm 0.8$ & 0.004 \\
& Self-stigma & $2.6 \pm 0.7$ & 0.001 \\
\hline Public stigma & Self-stigma & $\mathbf{0 . 3} \pm 0.6$ & $\mathbf{0 . 6 7 2}$
\end{tabular}

Bold italics show insignificant differences. secrecy were rated significantly higher than the other three factors, while the difference between treatment stigma and secrecy was inappreciable. Public stigma and self-stigma were the lowest rated factors, without a significant difference between the two. Perceived stigma differed significantly from all other stigma factors (Fig. 1). The mean differences in scores between individual stigma factors are depicted in Table 2.

\section{DEMOGRAPHics AND ForCE OF STIGMA FACTORS}

Secrecy correlated negatively with age (Fig. 2A; r = -0.322; $\mathrm{P}<0.05)$ and the level of education correlated positively with Secrecy (Fig. $2 \mathrm{~B} ; \tau=0.314$; $\mathrm{P}<0.01$ ), i.e., the older patients were less willing to display secrecy, but, interestingly, for better educated ones covering up their health problems seemed preferable.

Of note, the display of Secrecy and the perception of Self-stigma scored higher in the married than unmarried patients; $16.8 \pm 1.1$ vs. $11.9 \pm 1.0(\mathrm{P}<0.01)$ and $10.7 \pm 1.1$ vs. $7.8 \pm 0.8$ points $(\mathrm{P}<0.05)$, respectively. Secrecy and Treatment stigma were also higher in the subjects living with significant others than those living lonely; $15.9 \pm 0.9$ vs. $10.9 \pm 1.1(\mathrm{P}<0.01)$ and $15.5 \pm 0.9$ vs. $12.4 \pm 1.3$ points $(\mathrm{P}<0.05)$, respectively. Patients who lived lonely (aged $60.3 \pm 2.5$ years) were appreciable older than those living with families (aged 52.1 \pm 1.7 years $)(\mathrm{P}<0.05)$.

\section{Personality AND Stigma FACTORS}

Pearson's analysis showed significant correlations between all stigma factors, except for Public stigma, and neuroticism. These correlations are exemplified graphically for Treatment stigma and Perceived stigma vs. neuroticism in Fig. 3 (Panel A and B, respectively). Perceived stigma, Public stigma, and Self-stigma were related to extroversion. The only stigma factors related to both personality factors, i.e., neuroticism and extroversion, were Perceived stigma and Self-stigma. The associations between personality and stigma factors are depicted in Table 3.

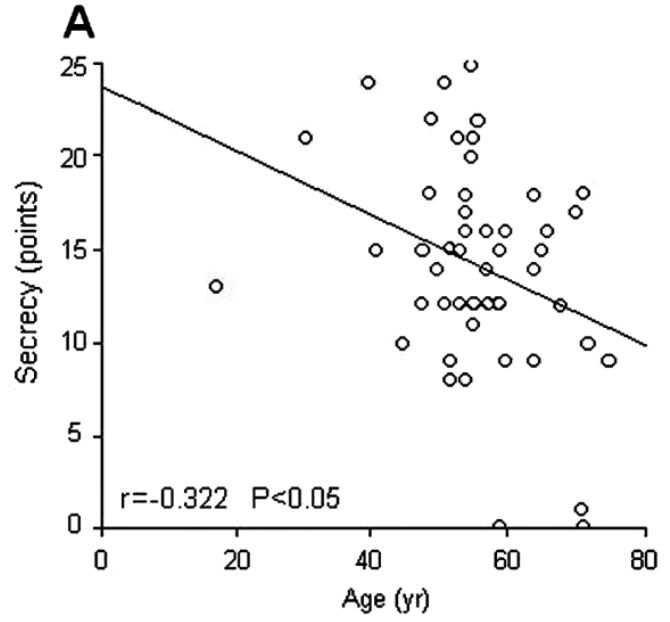

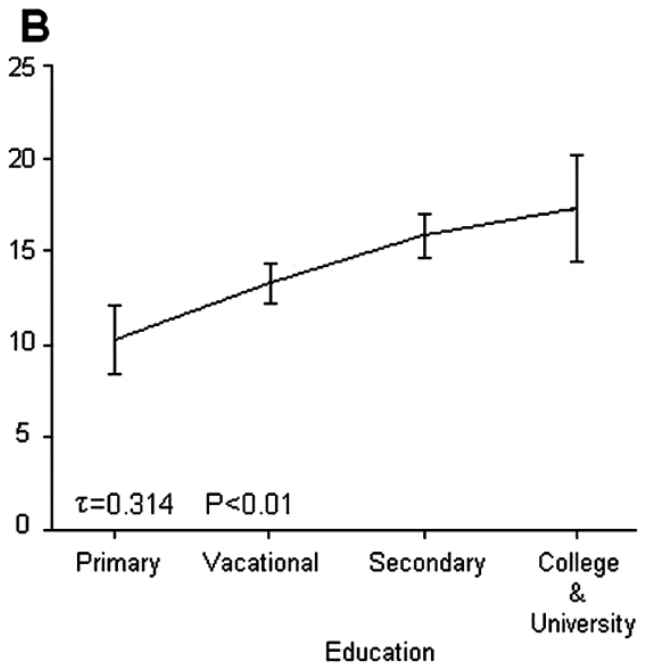

Fig. 2. Dependence of the level of Secrecy on age (Panel A) and on education (Panel B) of depressive patients. 
A

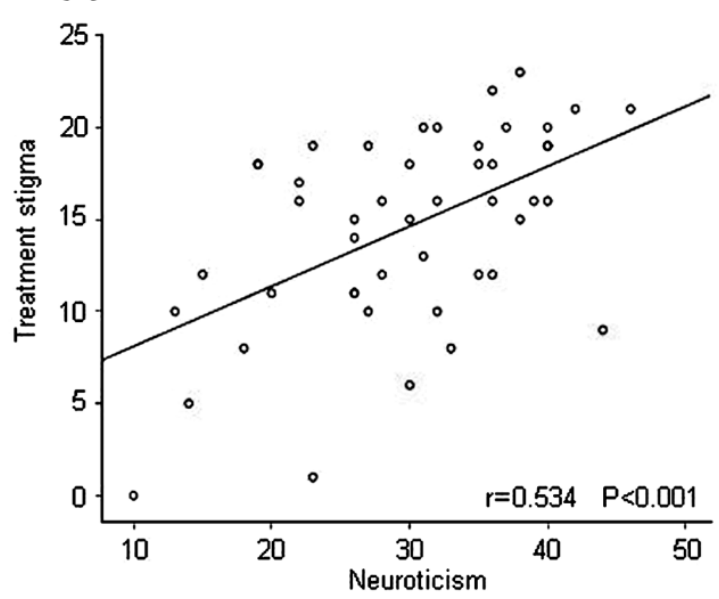

B

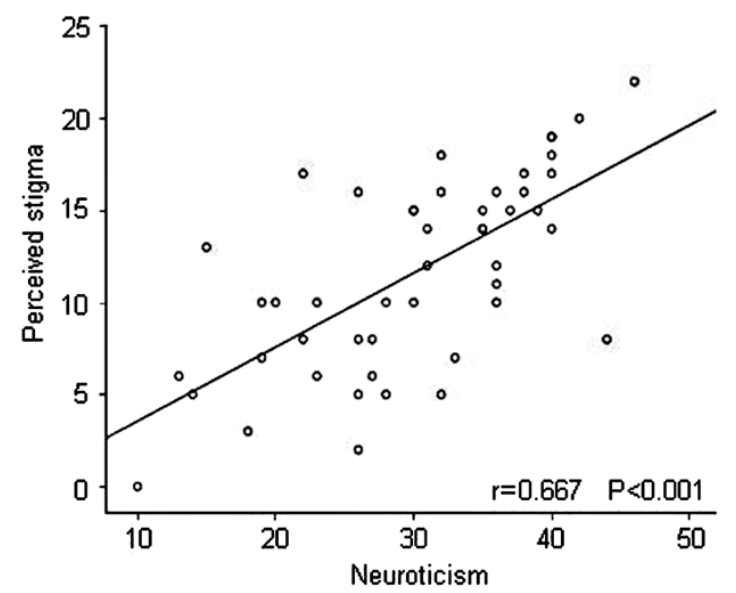

Fig. 3. Correlations between Treatment stigma (Panel A) and Perceived stigma (Panel B) and neuroticism.

Table 3. Personality and stigma factors.

\begin{tabular}{llll}
\hline & & Neuroticism & Extroversion \\
\hline Treatment stigma & $\mathrm{r}$ & 0.534 & -0.151 \\
& $\mathrm{P}$ & 0.001 & 0.306 \\
Secrecy & $\mathrm{r}$ & 0.368 & -0.243 \\
& $\mathrm{P}$ & 0.010 & 0.095 \\
Perceived stigma & $\mathrm{r}$ & 0.667 & -0.331 \\
& $\mathrm{P}$ & 0.001 & 0.022 \\
Public stigma & $\mathrm{r}$ & 0.142 & -0.339 \\
& $\mathrm{P}$ & 0.337 & 0.018 \\
Self-stigma & $\mathrm{r}$ & 0.357 & -0.332 \\
& $\mathrm{P}$ & 0.013 & 0.021 \\
\hline
\end{tabular}

\section{Depression STIGMA QUESTIONNAIRE (DSQ) -} PSYCHOMETRICAL VALIDITY

Analysis of the scores of the stigma subscales of the DSQ tallied in the main part of the study, in which depressive patients rated their perceived stigmatization, brought out a number of interrelationships which con- firm the DSQ's psychometrical validity, and which had not been found in the questionnaire results during the preliminary validation. All subscales significantly correlated with one another, except for the pair secrecy and public stigma (Table 4). In addition, all subscales correlated with the results of Beck's Depression Inventory (Table 5).

Table 5. Depression Stigma Questionnaire (DSQ) subscales and Beck's Depression Inventory.

\begin{tabular}{llc}
\hline & & Depression Inventory \\
\hline Treatment stigma & r & 0.650 \\
& P & 0.001 \\
Secrecy & r & 0.436 \\
Perceived stigma & P & 0.002 \\
Public stigma & P & 0.718 \\
& r & 0.001 \\
Self-stigma & P & 0.385 \\
& r & 0.007 \\
& P & 0.483 \\
\hline
\end{tabular}

Table 4. Correlations between subscales of Depression Stigma Questionnaire (DSQ).

\begin{tabular}{|c|c|c|c|c|c|c|}
\hline & & Treatment & Secrecy & Perceived & Public stigma & Self-stigma \\
\hline \multirow[t]{2}{*}{ Treatment stigma } & $\mathrm{r}$ & 1 & 0.668 & 0.718 & 0.362 & 0.513 \\
\hline & $\mathrm{P}$ & - & 0.001 & 0.001 & 0.012 & 0.001 \\
\hline \multirow[t]{2}{*}{ Secrecy } & $\mathrm{r}$ & 0.668 & 1 & 0.395 & 0.158 & 0.333 \\
\hline & $\mathrm{P}$ & 0.001 & - & 0.005 & 0.282 & 0.021 \\
\hline \multirow{2}{*}{ Perceived stigma } & $\mathrm{r}$ & 0.718 & 0.395 & 1 & 0.335 & 0.549 \\
\hline & $\mathrm{P}$ & 0.001 & 0.005 & - & 0.020 & 0.001 \\
\hline \multirow[t]{2}{*}{ Public stigma } & $\mathrm{r}$ & 0.362 & 0.158 & 0.335 & 1 & 0.502 \\
\hline & $\mathrm{P}$ & 0.012 & 0.282 & 0.020 & - & 0.001 \\
\hline \multirow[t]{2}{*}{ Self-stigma } & $\mathrm{r}$ & 0.513 & 0.333 & 0.549 & 0.502 & 1 \\
\hline & $\mathrm{P}$ & 0.001 & 0.021 & 0.001 & 0.001 & - \\
\hline
\end{tabular}




\section{DisCUSSION}

In the present study we attempted to determine whether perception of social stigma by persons suffering from depressive disorders could have to do with the personality traits. We addressed the issue by studying linkage between neuroticism and extroversion, on the one side, and five basic dimensions of social stigma, such as self-stigma, perceived stigma, public or mirrored stigma, secrecy, and treatment stigma in depressive in-patients.

The major findings of this study were that treatment stigma and secrecy were the most frequent aspects of stigma experienced by the patients, neuroticism fostered the perception of secrecy, treatment-, perceived-, and self-stigma, while extroversion those of perceived-, self-, and mirrored-stigma. We also found that secrecy was negatively associated with age and positively with the level of education. Thus, the findings of the study draw attention to the relevance of personality resources in the way the depressive patients perceive and are able to cope with stigma label.

\section{ForCE OF STIGMA FACTORS}

A conspicuously high level of the sense of treatmentstigma may have to do with strong stereotypes, prejudices, and discrimination against psychiatric patients in general [12]. While other scales of the depression stigma questionnaire refer to depression itself, treatmentstigma is the only one referring to receiving treatment for depression. The noteworthy comparison here is the one between treatment- and perceived-stigma, since both reflect negative social attitudes concerning depression (other scales concern the coping style, subject's attitudes, and self-assessment), but perceived-stigma includes no references to treatment. Both these dimensions of stigma were heavily experienced by the patients, which speaks to the substantial negative social attitudes directed to, and perceived by, the patients. Taking into account that all of the patients were hospitalized in psychiatric hospitals during the study, it is understandable they referred to the psychiatric treatment. Thus, the outcome may be simply a reflection of low mental health literacy in the patients' social environment, one of the causes of existing prejudices and discrimination against psychiatric patients regardless of disorder, which has been previously reported by some other studies [13]. Although this is a hypothetical explanation, it is possible that a bad reception of psychiatric treatment perceived by patients is a factor contributing to regression of the patients' state and recovery. An interesting question arises of whether the place of treatment, be it a hospital or an outpatient ambulatory, makes a difference in the stigma perceived. Some studies have attempted to tackle this issue. A Belgian study [14] has demonstrated that being treated in a psychiatric hospital generates more stigma than that stemming from treatment in a general hospital. There is also evidence that the experienced discrimination due to a mental illness is not appreciably different in patients with different mental illnesses [15].

In the light of these findings, a high level of secrecy is not surprising, as it may be an avoidant strategy of coping, likely to be frequently used by people with limited stress coping resources, such as depressive patients. However, there are two interesting relationships; perhaps worthy of broader explorations, i.e., a decreasing frequency of secrecy in elderly patients and increasing frequency of secrecy in educated patients. The lower secrecy in the elderly may be explicable in terms of forming a specific psycho-social identity, being independent from social opinions, and reducing social contacts to people of about the same age range and with similar health problems. It is also possible that avoiding secrecy is part of a coping strategy included in the therapy plan. Health problems are one of few areas in which older patients choose active strategies of coping more frequently than other age-groups [16]. Active coping with social situations associated with depression is a strategy often suggested by therapists, and elderly patients, as more compliant than others, may simply obey it more often. Another explanation of lower secrecy with advancing age may has to do with the fact that older patients more often do not live with 'significant others'. Therefore, patients hide the fact of being treated for depression when there is someone 'worth' hiding it from; the issue of no concern to the lonely ones.

A high level of secrecy in well educated patients may be of more complex nature. At first glance, one could surmise a rather opposite dependence, because stereotypes, prejudices, and discrimination against psychiatric patients are lower (17), and task-oriented strategies (18) and compliance (19) are higher among well educated people. Also, well educated depressive patients declare a higher life quality. Simultaneously, in Poland these people are more afraid of being mentally ill and perceive the community of their peers as more stigmatizing [20]. These findings and some sociological theories may suggest that a decisive factor here is a fear of losing one's social status. In opposition to declared tolerance to psychiatric treatment, real attitudes held by well educated and active professionals may be less lenient, especially when one's social status is highly associated with intellectual performance and ability. Therefore, revealing a depressive condition may influence one's image of competency and efficiency, and generate fear of being socially devalued and loosing social status, which often is associated with mental illness [12]. This explanation remains intuitive, as no clear relationships were unraveled in the present study between the level of education and other stigma factors. Education, income, and occupation are the determinants of social status, which makes the hypothesis of their plausibly essential role in the psychoemotional way of perception of illness, treatment stigma, and coping strategies not unreasonable.

\section{Stigma And Personality Factors}

Strong relationships between neuroticism and all stigma factors, except for public stigma, confirm the hypothesis of the role of personal stress coping resources in the perception of stigma. This role does not seem undermined by less dedicated results obtained in the present study concerning the relation- 
ships between extroversion and stigma factors. Two of the five stigma factors estimated, treatment stigma and secrecy, did not relate significantly to both personality traits. The reason for the lack of relation may be due to the origin of stigma factors investigated in the study. Perceived- and self-stigma are constructs brought by Corrigan et al [21] as part of his stigma theory. They have been verified in various empirical studies by the authors and their associations with personality (i.e. self-esteem and self-efficacy) are confirmed. The other stigma factors are derived from the study of Kanter et al [11] and are based on the literature review, available stigma measures, and factorial analysis of questions. In particular, public or mirrored-stigma is a new notion in stigma research and has not yet been broadly investigated before. Mirrored-stigma refers to negative attitudes held by the subject against his own group. It may be a defensive mechanism against stigma based on projection, denial, or otherwise, facilitating the maintenance of self-esteem through self-enhancement. The exact nature of this factor is unknown and it is necessary to conduct a basal investigation of its theoretical background and empirical characteristics.

In the present study, we found that, in general, relationships between neuroticism and stigma factors were higher than those between extroversion and stigma factors. That is probably due to the fact that neuroticism contributes more to depression and to stresscoping strategies than extroversion does.

\section{Contribution to Depression Stigma Questionnaire (DSQ)}

The study brought new information about DSQ validity. The correlations between DSQ subscales, describing the factors of treatment stigma, secrecy, perceived stigma, public or mirrored stigma, and self-stigma, which were not present in the validation part of the study, showed up in the main experimental part of it. All subscales correlated with one another, which contributes to, and strengthens, the internal accuracy of the whole DSQ and the accuracy of particular subscales. Correlations between all subscales and depressive symptoms bring an additional contribution to the accuracy of DSQ, as they reaffirm that stigma is related to depression.

\section{Practical Implications and FURTHER InVESTIGATORY} ISSUES

It is already evident that varied factors of stigma in the setting of schizophrenia have an influence on the outcome of, or can even predict, the patients' performance, such as low adherence to treatment or its discontinuation; the effect possibly being moderated by psychological factors, such as personality. Stigma also has an impact on patients' employment or habitation problems, and can have a host of other unsettling psycho-social ramifications. It is thus noteworthy to analyze whether like problems are of significant meaning in depressive patients as well. Particularly, the role of hospitalization in the perception of stigma should be taken into consideration, let alone demographical and individual patients' differences. Further study designs should then focus on investigating the roles of entwined interrelationships between the factors mentioned above, exploring the nature of stigma in depression and constructing useful assessment tools to enable to compare stigmatization in different pathological states.

In conclusion, the present study shows that depression is a highly stigmatized illness and confirms the relevance of personality resources in the perception and coping with stigma. The study also points out the importance of hospitalization and secrecy as factors in the stigmatization process. In light of the present findings, stigma experienced by a depressive patient is not only related to the external indicators of social stigmatization and the state of depression, but also to the personal psychoemotional features of an individual. We believe we have shown the possible psychological background of individual differences in stigma perception and coping with it, which may be of help in attempts to restore the patients' social role and wellness.

Conflicts of interest: No conflicts of interests were declared by the authors in relation to this article.

\section{REFERENCES}

1. Ottati V, Bodenhausen GV, Newman L. Social psychological models of mental illness stigma. In: Corrigan PW (ed.). On the Stigma of Mental Illness. Practical Strategies for Research and Social Change. American Psychological Association: Washington, DC, 2005; p. 100.

2. Crisp AH, Gelder MG, Rix S, Meltzer HI, Rowlands OJ. Stigmatisation of people with mental illnesses. B J Psychiatry 2000; 177: 4-7.

3. Weiner B. On sin versus sickness. A theory of perceived responsibility and social motivation. Am Psychol 1993; 48(9): 957-65.

4. Hinshaw SP. The Mark of Shame: Stigma of Mental Illness and an Agenda for Change. Oxford University Press: US, 2007.

5. Corrigan PW, Kleinlein P. The impact of mental illness stigma. In: Corrigan PW (ed.) On the Stigma of Mental Illness. Practical Strategies for Research and Social Change. American Psychological Association: Washington, DC, 2005; p. 27.

6. Sirey JA, Bruce ML, Alexopoulos GS, Perlick DA, Friedman SJ, Meyers BS. Stigma as a barrier to recovery: Perceived stigma and patient rated severity of illness as predictors of antidepressant drug adherence. Psychiatr Serv 2001; 52(12): 1615-20.

7. Sirey JA, Bruce ML, Alexopoulos GS, Perlick DA, Raue P, Friedman SJ, Meyers BS. Perceived stigma as a predictor of treatment discontinuation in young and older outpatients with depression. Am J Psychiatry 2001; 158(3): 47981.

8. Crocker J, Major B. Social stigma and self esteem: The self protective properties of stigma. Psychol Rev 1989; 96(4): 608-30.

9. Miller CT, Major B, Coping with stigma and prejudice. In: Heatherton TF, Kleck RE, Hebl MR, Hull JG (eds.) The Social Psychology of Stigma. Guilford Press: New York, 2003.

10. Zawadzki B, Strelau J, Szczepanik P, Sliwinska M. Polish Textbook of Costa and McCrae's NEO-FFI Inventory. Laboratory of Psychological Tests. The Polish Psychological Society: Warsaw, 1998. 
11. Kanter JW, Rusch LC, Brondino MJ. Depression self-stigma. A new measure and preliminary findings. J Nerv Ment Dis 2008; 196(9): 663-70.

12. Goffman E, Stigma. Notes on the Management of Spoiled Identity. Touchstone, 1986.

13. Romer D, Bock M. Reducing the stigma of mental illness among adolescents and young adults: The effects of treatment information. J Health Commun 2008; 13(8): 742-58.

14. Verhaeghe M, Bracke P, Bruynooghe P. Stigmatization in different mental health services: a comparison of psychiatric and general hospitals. J Behav Health Serv Res 2007; 34(2): 186-97.

15. Feldman D, Crandall C. Dimensions of mental illness stigma: What about mental illness causes social rejection? J Soc Clin Psychol 2007; 26(2): 137-54.

16. Ngoh LL, Health literacy: A barrier to pharmacist-patient communication and medication adherence. Pharmacy Today 2009; 15(8): 45-57.

17. Dyduch A, Grzywa A. Stigma and related factors basing on mental illness stigma. Pol Merkur Lekarski 2009; 26(153): 263-7.

18. Ngoh LL. Health literacy. A barrier to pharmacist-patient communication and medication adherence. Pharmacy Today 2009; 15(8): 45-57.
19. Orzechowska A, Florkowski A, Gruszczynski W, Zboralski K, Wysokinski A, Galecki P, Talarowska M. Socio-economic status, aggressive behaviours and coping with stress. Psychiatr Pol 2009; 43(1): 53-63.

20. Mental illnesses. Social stereotype and distance: a report. Public Opinion Research Center (CBOS), 2000; Available from: www.bezuprzedzen.org/doc/06Choroby_psychi cznestereotyp_i_dystans_2000_CBOS.pdf; Accessed May 19, 2010.

21. Corrigan P, Watson A, Barr L. The self-stigma of mental illness: implications for self-esteem and self-efficacy. J Soc Clin Psychol 2006; 25(8): 875-84.

Address for correspondence:

M. Pokorski

Medical Research Center

Polish Academy of Sciences

5, Pawinskiego St.

02-206 Warsaw

Poland

Phone/Fax: +48 226086622

E-mail: m_pokorski@hotmail.com 